\title{
MÚSICA Y DESCARGA DE INTERNET: ANÁLISIS DE SUS ANTECEDENTES E IMPLICACIONES DE GESTIÓN
}

\section{Marketing de la Cultura}

\author{
MANUEL CUADRADO GARCÍA \\ Universitat de València, España \\ manuel.cuadrado@uv.es \\ MARÍA JOSÉ MIQUEL ROMERO \\ Universitat de València, España \\ maria.j.miquel@uv.es
}

\section{RESUMEN}

La descarga de música de Internet es un fenómeno cada vez más extendido, que está causando importantes daños a la industria musical. Tomando como referencia la Teoría del Comportamiento Planeado, el presente trabajo trata de identificar las variables que condicionan la intención del individuo de descargarse música no autorizada, incorporando nuevas relaciones no contempladas explícitamente en la teoría considerada. Sólo conociendo el por qué del comportamiento se podrá valorar la posible instrumentalización de acciones a tendentes mejorar la situación por la que atraviesa la industria musical. A partir de los resultados obtenidos, se proponen acciones vinculadas con la gestión.

PALABRAS CLAVE: ACTITUD, NORMA SUBJETIVA, PIRATERÍA MUSICAL, TEORÍA DEL COMPORTAMIENTO PLANEADO.

\section{ABSTRACT}

Internet music downloading is a widespread activity which is causing important loses to the music industry. Taking Planned Behaviour Theory as a reference, this article identifies the variables that determine the intention of individual music downloading and incorporates new relationships not considered explicitly in the mentioned theory. Only by acknowledging the factors that motivates that behaviour, it will be possible to propose managerial actions trying to improve the situation of the music industry. According to the results, management actions are proposed.
KEY WORDS: ATTITUDE, SUBJECTIVE NORM, MUSIC PIRACY, THEORY OF PLANNED BEHAVIOUR.

\section{INTRODUCCIÓN}

No cabe duda que la tecnología está hoy presente en todas las facetas del día a día, tanto de la sociedad en general, como del negocio empresarial en particular. A pesar de las enormes ventajas que ello reporta, no hay que olvidar que precisamente las rápidas conexiones a Internet, la disponibilidad de alta capacidad de almacenaje a un coste mínimo, junto con las redes peer-to-peer de Internet, imposibles de controlar, han dejado las puertas abiertas a la piratería. Software, música, películas o libros no escapan al lado oscuro de la tecnología. Cronan y Al-Rafee (2008) califican a este tipo de piratería como piratería digital, y la definen como la copia o descarga ilegal de software y archivos (media files) con derechos de autor. En medio de la polémica por aprobar una ley en España que penalice la disposición gratuita de productos protegidos por derechos de autor, a través de la descarga de archivos de redes descentralizadas en Internet (P2P) el aumento del consumo de estos productos por dicha vía y otras nuevas de naturaleza digital (iTunes, Spotify, teléfonos móviles, etc.) es una realidad a nivel global.

El sector de la música es actualmente un negocio global que contribuye al Producto Interior Bruto de un país y genera empleo. Un sector 
que el año 2009 generó unas ventas de más de 25400 millones de dólares en España (SGAE, 2010). Sin embargo, la aparición de Internet, archivos digitales, y programas de descarga de música, le han afectado enormemente. Aunque existen numerosos efectos positivos, como la mayor facilidad para dar a conocer grupos de música, o recuperar aquellos caídos en el olvido, son mayoritarios los efectos negativos con los que se asocia. En este sentido, según datos del anuario SGAE (2010), en España, de cada cien canciones que se descargan, 96 llegan a su destinatario de manera ilegal, lo que supone unas pérdidas de 1300 millones de euros.

Esta situación conlleva la necesidad de un cambio de modelo de gestión en la industria de la música, replanteando sus formas de distribución y venta. En este contexto, la literatura sugiere el desarrollo de investigaciones que analicen las decisiones del consumidor, con el objetivo de definir mejor las acciones a desarrollar. Las enormes pérdidas que esta forma de piratería está generando plantean la necesidad de entender mejor por qué ocurre este comportamiento. Los factores tecnológicos que lo hacen posible mejoran constantemente y forman parte de la vida cotidiana de una gran cantidad de individuos; por ello, resulta fundamental conocer qué es lo que lleva a desarrollar este tipo de comportamientos para valorar si es posible desarrollar algún tipo de actuación que consiga frenarlos, buscando a la vez nuevas formas de revitalizar la industria musical.

El objetivo del presente trabajo se centra de forma exclusiva en la descarga de música no autorizada de Internet. Nuestro propósito es analizar los antecedentes de la intención de descarga de música en el marco de la Teoría del Comportamiento Planeado (Fishbein y Ajzen, 1975; Ajzen y Fishbein, 1980), cuya principal premisa es que la intención es el mejor predictor del comporta- miento. A partir de los resultados se plantean propuestas de acción para la industria de la música.

\section{REVISIÓN DE LA LITERATURA}

\section{La teoría del comportamiento planeado}

La Teoría de la Acción Razonada (Fishbein y Ajzen, 1975; Ajzen y Fishbein, 1980) y su extensión, la Teoría del Comportamiento Planeado (Ajzen, 1985,1991 ) han sido ampliamente utilizadas en las últimas dos décadas, y aún en la actualidad, para analizar toda una variedad de intenciones de comportamiento y comportamientos en sí. En concreto, su aportación ha sido especialmente valiosa en el estudio de conductas en el ámbito de la ética (e.g. Cronan y Al-Rafee, 2008; Bailey, 2006; Chiu, 2003; Tonglet, 2002; Flannery y May, 2000; Parker et al. 1992; Dubinsky y Loken, 1989).

La Teoría del Comportamiento Planeado (Ajzen, 1985,1991 ) (TCP de ahora en adelante) fue desarrollada como una extensión a la Teoría de la Acción Razonada de Ajzen y Fishbein (1980) (TAR a partir de ahora). Según ambas teorías, la clave para predecir un determinado comportamiento descansa en las intenciones de desarrollar dicho comportamiento. La TAR plantea que el comportamiento humano es bastante racional, dado que el individuo considera las consecuencias de su comportamiento antes de desarrollarlo; de acuerdo con la TAR, son dos los principales determinantes que afectan a la conducta humana, uno de naturaleza personal, la actitud hacia dicho comportamiento, y otro de naturaleza más social, como es la norma subjetiva. Esta última variable identifica las percepciones que posee el individuo sobre lo que pensaría la gente que le importa (familia, amigos, compañeros,...), si él/ ella desarrollase o no el mencionado comportamiento (Ajzen, 1985). 
Adicionalmente, la actitud hacia el comportamiento es función de las creencias que posea el individuo de que dicho comportamiento dará ciertos resultados, así como también, de la valoración de los resultados del comportamiento. Por su parte, la norma subjetiva quedaría conformada por las creencias normativas sobre lo que otras personas importantes para el individuo opinan de dicho comportamiento, y por la motivación por complacer del individuo a esas personas importantes.

La TCP (Ajzen, 1985, 1991) toma como base la TAR, pero identifica que no es adecuada para explicar comportamientos en situaciones en las que dicha conducta no está bajo el control del individuo. De esta forma, la TCP identifica otro factor determinante de la intención de comportamiento, adicional a los recogidos en la TAR: el control percibido del comportamiento. Esta variable, fácilmente medible, recoge las creencias individuales sobre lo fácil o difícil que puede resultar el desarrollo de la conducta (Ajzen y Madden, 1986).

\section{La investigación sobre piratería musical y su conexión con la tcp: planteamiento de hipótesis}

El marco de referencia que ha nutrido los inicios de la investigación sobre piratería musical descansa en las anteriores y prolíficas investigaciones desarrolladas sobre piratería de software. Tanto la piratería musical como la de software son consideradas faltas leves, al atentar "solamente" contra la propiedad intelectual (Gupta, Gould y Pola, 2004; Logsdon et al. 1994). Esta similitud es la que ha llevado a utilizar los resultados de la gran mayoría de investigaciones sobre la piratería de software al plano de la piratería musical, con el propósito de entender su naturaleza (d'Astous, Colbert y Montpetit, 2005). No obstan- te, hay estudios que han demostrado ciertas diferencias entre ambos comportamientos (Gopal, Sanders, Bhattacharjee, Agrawal, Wagner, 2004). Aunque cada vez es más abundante la investigación relacionada con la piratería musical, queda todavía lejos de ser, en volumen, similar a la desarrollada en relación a la piratería de software. Y aún más: en toda la revisión bibliográfica realizada, tan sólo se han encontrado tres estudios que modelicen y establezcan relaciones entre la actitud hacia la piratería musical, la intención de piratear música y sus antecedentes.

En el año 2005 se publican los dos primeros estudios centrados exclusivamente en la piratería musical, y que modelizan algunas de las relaciones identificadas en la TCP (D'Astous, Colbert y Montpetit, 2005; Chiou, Haung y Lee, 2005). D'Astous et al. (2005) identifican como antecedentes de la actitud hacia la piratería tres variables: el comportamiento pasado, las consecuencias personales negativas y la predisposición ética, siendo también tres los antecedentes de la intención de piratear: la actitud hacia la piratería musical, la norma subjetiva y el control percibido del comportamiento. Por su parte, Chiou et al (2005), quienes explícitamente no consideran la TCP como marco de su trabajo, amplían el número y cambian los antecedentes de la actitud hacia la piratería: la idolatría que se tenga hacia el cantante o grupo, la satisfacción obtenida, el riesgo de ser atrapado, la percepción de la magnitud de las consecuencias, la percepción del consenso social y la proximidad percibida; y también cambian los antecedentes de la intención de piratear: actitud hacia la piratería, idolatría hacia el cantante o grupo y proximidad percibida. La investigación más reciente de Cronan y Al-Raffe (2008), aunque en el ámbito más general de la piratería digital (en la que se incluye la música), sí que plantea explícitamente una ampliación de la TCP añadiendo la variable obligación moral como antecedente de la 
intención; esta "ampliación" es posible gracias a la flexibilidad de la TCP que permite considerar otras variables teóricas relevantes para explicar un comportamiento (Ajzen, 1991). No obstante, Cronan y Al-Rafee (2008) no consideran en su estudio los antecedentes de la actitud hacia la piratería. De acuerdo con estos estudios y según plantea la premisa básica de la TCP, plateamos la primera hipótesis que relaciona actitud con intención de comportamiento:

H1.- Existe una relación positiva entre la actitud hacia la descarga de música no autorizada de Internet y la intención de descargar música.

En el marco de la TCP, la actitud hacia el comportamiento es función de las creencias que posea el individuo de que dicho comportamiento dará ciertos resultados, así como también, de la valoración de los resultados del comportamiento. En lo que hace referencia a la relación consecuencias del comportamiento y actitud, sólo el estudio de Chiou et al (2005) analiza cómo percibe el individuo las consecuencias de la piratería musical (compra no original, descarga o copia no autorizada) sobre distintos implicados en la industria musical y la sociedad en general, constatándose dicha relación. En el trabajo de D'Astous et al. (2005), sin embargo, lo que se analizan son las consecuencias negativas personales (riesgo de multa,...); si tenemos en cuenta el objeto de estudio (la descarga personal de música no autorizada de Internet), creemos que el individuo no percibe consecuencias negativas para él. Todo lo contrario, y acogiéndonos a la TCP, lo que percibirá realmente a nivel personal son beneficios. Por ello, planteamos que:

H2.- Existe una relación negativa entre las creencias que posea el individuo sobre las consecuencias negativas de la descarga de música no autorizada y la actitud hacia la descarga de música.
H3.- Existe una relación positiva entre la valoración que atribuya el individuo a los beneficios de la descarga de música no autorizada y la actitud hacia la descarga de música

Haciendo uso de esa flexibilidad de la TCP que apunta Ajzen (1991) para incorporar nuevas variables, consideramos fundamental incluir en el modelo la importancia que tienen para el individuo a nivel personal, las consecuencias negativas de la descarga de música en el ámbito de la industria musical; al ser consecuencias no soportadas en un entorno cercano, el individuo puede verse más o menos implicado con ellas. Esta valoración es identificada en el trabajo de Chiou et al (2005) como proximidad percibida. Por tanto:

H4.- Existe una relación negativa entre la importancia que a nivel personal le atribuya el individuo a las consecuencias negativas de la descarga de música no autorizada, y la actitud hacia la descarga de música.

Un segundo antecedente de la intención de comportamiento en la TCP es la norma subjetiva. Esta variable ha sido medida y relacionada de forma muy distinta en los estudios previamente mencionados. En el trabajo de Chiou et al. (2005) se la denomina consenso social percibido, y aparece relacionada con la actitud, no con la intención; además, sólo identifica la percepción que tiene el individuo sobre lo que los demás opinan en relación a la piratería musical; no recoge, pues, la motivación por complacer identificada en la TCP; por el contrario, el de D'Astous et al. (2005) y el de Cronan y Al-Rafee (2008) la relaciona con la intención, y sólo recoge la motivación por complacer (Cronan y Al-Rafee, 2008) o una aproximación (D'Astous et al., 2005). En nuestro estudio hemos analizado sólo la percepción de la opinión de los demás sobre la descarga de música: si consideramos, como ha sido constatado, que la descarga de música de Internet es un comportamiento socialmente aceptado, la 
motivación por complacer en relación a dicho comportamiento consideramos que no será especialmente relevante. A partir de estos resultados y reflexiones proponemos las siguientes hipótesis:

H5.- Existe una relación negativa entre la percepción que posea el individuo sobre la opinión negativa de los demás en relación a la descarga de música no autorizada de Internet (norma subjetiva), y la actitud hacia la descarga de música.

H6.- Existe una relación negativa entre la percepción que posea el individuo sobre la opinión negativa de los demás en relación a la descarga de música no autorizada de Internet (norma subjetiva), y la intención de descargar música.

En último lugar, también directamente de la TCP se desprende la relación directa entre la percepción sobre poseer las habilidades necesarias para desarrollar un comportamiento y la intención en sí de desarrollarlo. Esta relación ha sido ya contrastada en la investigación sobre piratería musical (D'Astous et al., 2005; Cronan y Al-Rafee, 2008). No obstante, creemos importante, además, incluir en el modelo la disponibilidad de capacidad técnica, dado que es una condición necesaria, aunque no suficiente, para desarrollar el comportamiento en cuestión. En base a esto, planteamos las dos últimas hipótesis:

H7.- Existe una relación positiva entre la percepción que tenga el individuo de que posee las habilidades necesarias para descargar música no autorizada de Internet, y la intención de descargar música.

H8.- Existe una relación positiva entre la disponibilidad técnica que posea el individuo para descargar música no autorizada de Internet, ya la intención de descargar música.

Las relaciones hipotetizadas se reflejan en la figura 1.

\section{METODOLOGÍA}

Los datos para contrastar las hipótesis planteadas, mediante ecuaciones estructurales, provienen de una investigación centrada en el estudio de consumidores de música. El método de recogida de información utilizado fue la entrevista personal con cuestionario estructurado. El universo considerado fueron individuos de 15 a 45 años residentes en la provincia de Valencia. El hecho de acotar dicho tramo de edad viene justificado por ser este grupo de población el de mayor disponibilidad y uso de las nuevas tecnologías de reproducción digital, frente al resto de la población, así como el de mayor consumo de música.

El método de muestreo seleccionado fue el de cuotas en base a la edad y al género, tratando de buscar una mayor representatividad. Tras depurar los datos, se consiguió una muestra válida de 1806 casos.

Las escalas de medida utilizadas tienen como punto de partida las investigaciones consultadas al respecto, especialmente los estudios de Chiou et al. (2005) y de d'Astous et al. (2005), también en línea con el trabajo de Cronan y Al-Rafee (2008). Todas las variables se midieron sobre escalas likert de cinco punto, a excepción de la capacidad técnica, que fue una escala de tipo sumativo. En concreto, la escala que medía la percepción sobre las consecuencias de la descarga de música, y la valoración de dichas consecuencias, por parte del individuo, se midieron cada una con cuatro ítems; la valoración de los beneficios que el individuo atribuye a la descarga de música se midió sobre una escala de nueve ítems; la actitud hacia la descarga de música se midió sobre una escala de cuatro ítems, y las habilidades con dos ítems. Las escalas intención de descargarse música no autorizada y capacidad técnica se constituyeron por un ítem cada una. 


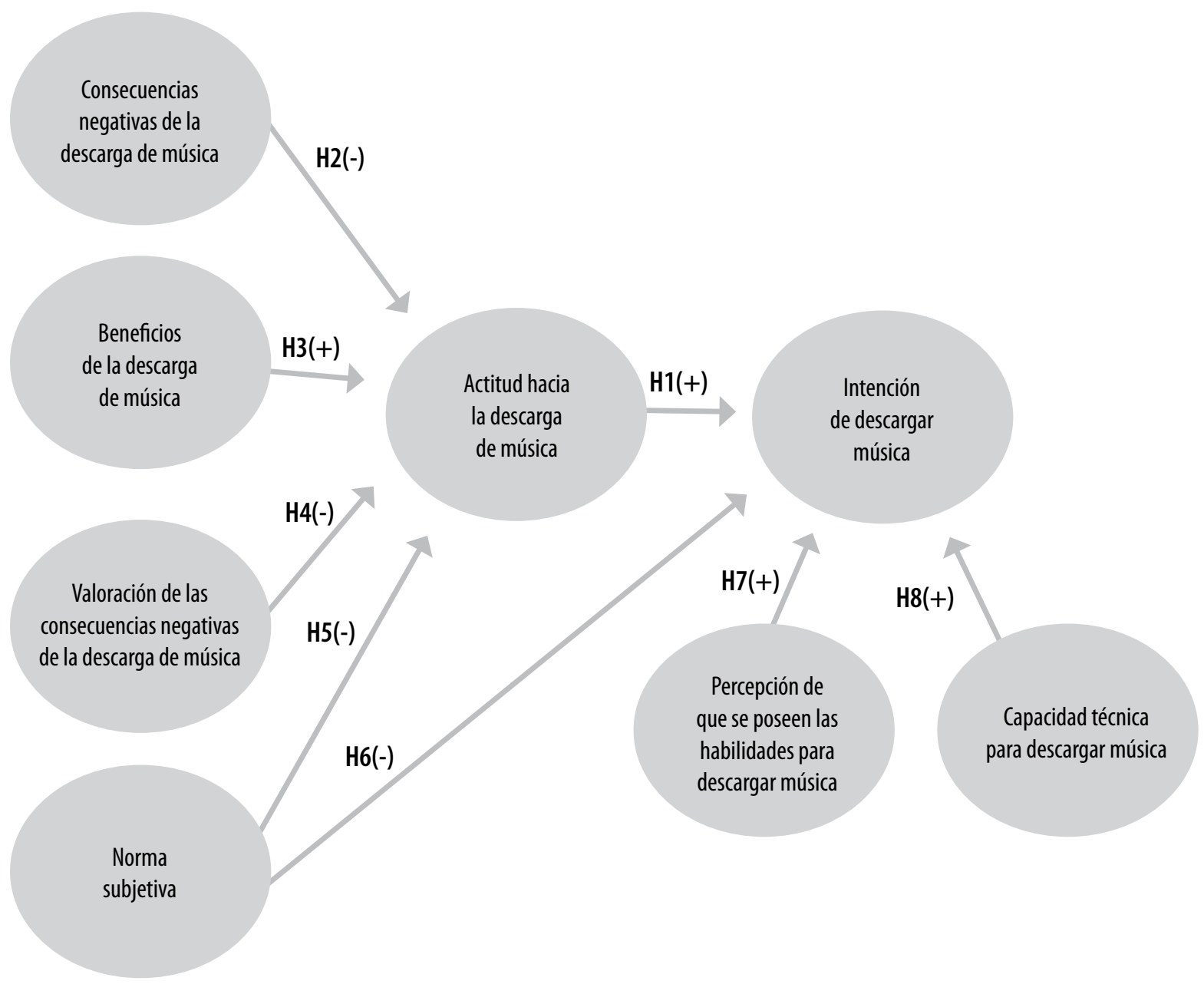

\section{RESULTADOS}

\section{Descriptivos}

Antes de abordar propiamente el contraste de hipótesis consideramos relevante analizar de forma breve las variables analizadas en el estudio, a nivel descriptivo (Tabla 1).

De los resultados descriptivos mostrados en la Tabla 1 se deduce que el individuo percibe lige- ramente consecuencias negativas de la descarga de música sobre el cantante y la discográfica, y no tanto sobre la sociedad en general, o la persona que compra música en formato original. No obstante, sí que le atribuyen importancia a nivel personal, aunque también de forma muy sutil, al impacto negativo de la descarga sobre el cantante. 
TABLA 1

\section{DESCRIPTIVOS ( 1 = TOTAL DESACUERD0/ 5 = TOTAL ACUERD0; CAPACIDAD TÉCNICA: ESCALA SUMATIVA)}

\begin{tabular}{|c|c|c|c|}
\hline & ITEMS & Media & Desv. Típ. \\
\hline \multirow{4}{*}{$\begin{array}{l}\text { PERCEPCIÓN CONSECUENCIAS } \\
\text { NEGATIVAS DE LA DESCARGA DE MÚSICA }\end{array}$} & Impacto en cantante & 3,36 & 1,203 \\
\hline & Impacto en discográfica & 3,63 & 1,140 \\
\hline & Impacto en sociedad & 2,55 & 1,072 \\
\hline & Impacto en comprador original & 2,62 & 1,198 \\
\hline \multirow{4}{*}{$\begin{array}{l}\text { VALORACIÓN CONSECUENCIAS NEGATIVAS } \\
\text { DE LA DESCARGA DE MÚSICA }\end{array}$} & Impacto en cantante no importa & 2,89 & 1,112 \\
\hline & Impacto en discográfica no importa & 3,47 & 1,153 \\
\hline & Impacto en sociedad no importa & 2,95 & 1,075 \\
\hline & Impacto en comprador original no importa & 3,14 & 1,143 \\
\hline \multirow{9}{*}{$\begin{array}{l}\text { BENEFICIOS PERCIBIDOS } \\
\text { DE LA DESCARGA DE MÚSICA }\end{array}$} & Es económico & 4,55 &, 729 \\
\hline & Tengo más música & 4,23 &, 934 \\
\hline & Hago mis álbumes & 3,91 & 1,131 \\
\hline & Cds originales muy caros & 4,35 &, 846 \\
\hline & Quiero escucharla antes & 3,23 & 1,200 \\
\hline & No está en tiendas & 2,38 & 1,283 \\
\hline & Es más cómodo & 3,94 & 1,107 \\
\hline & Sólo interesan ciertas canciones & 3,71 & 1,166 \\
\hline & La descarga ayuda a difundir música & 3,43 & 1,098 \\
\hline \multirow{3}{*}{ NORMA SUBJETIVA } & Gente considera descarga no correcto & 2,67 & 1,227 \\
\hline & Amigos desacuerdo yo descargue & 2,12 & 1,067 \\
\hline & Amigos creen descarga no ético & 2,30 & 1,112 \\
\hline \multirow{4}{*}{$\begin{array}{l}\text { ACTITUD HACIA } \\
\text { LA DESCARGA DE MUSICA }\end{array}$} & Me gusta idea de descargar música & 3,80 & 1,198 \\
\hline & Hoy no dudaría en descargar música & 3,87 & 1,242 \\
\hline & Prefiero música original & 2,41 & 1,197 \\
\hline & No hay nada malo en descargarse música & 3,81 & 1,198 \\
\hline \multirow{2}{*}{$\begin{array}{l}\text { HABILIDADES } \\
\text { PARA DESCARGAR }\end{array}$} & Tengo las habilidades & 3,44 & 1,521 \\
\hline & Conozco algún programa & 3,15 & 1,509 \\
\hline CAPACIDADTECNICA & Capacidad técnica & 4,21 & 2,218 \\
\hline INTENCIÓN DE DESCARGA & Próximamente tengo intención de descargar música & 3,64 & 1,411 \\
\hline
\end{tabular}

Fuente: elaboración propia. 
En relación a los beneficios percibidos de la descarga de música no autorizada de Internet, se constata que los beneficios económicos son los más percibidos, seguido de los beneficios relacionados con la comodidad que implica el hecho de descargarse música de Internet. Además, el individuo considera que los demás (público en general y amigos) no creen que la descarga de música sea algo incorrecto o poco ético. En esta línea, los ítems que configuran la variable actitud hacia la descarga de música no autorizada ponen de manifiesto que la actitud hacia tal comportamiento es positiva, igual como la intención de descargar. Esta situación viene acompañada por una capacidad técnica adecuada para descargar música, y la percepción de poseer las habilidades necesarias.

\section{Propiedades psicométricas y contraste de hipótesis}

Para proceder a contrastar las hipótesis planteadas fue necesaria la validación previa de los instrumentos de medida utilizados, comprobándose las principales propiedades psicométricas de los mismos.

En este sentido, la fiabilidad se valoró a partir del alpha de Cronbach (Cronbach, 1951) y de la correlación ítem-total (Bagozzi, 1981) para cada una de las escalas. El análisis del alpha de Cronbach eliminando un elemento no llevó a descartar ningún ítem, pues todos superaban el umbral mínimo de 0,3 (Norusis, 1993). El alpha de Cronbach para cada una de las escalas utilizadas mostró resultados satisfactorios ya que en todos los casos presentó valores superiores a 0,7 (Nunnally, 1978).
Para contrastar la validez convergente de las escalas se realizó un análisis factorial confirmatorio sobre las seis variables latentes (consecuencias del comportamiento, valoración de las consecuencias, valoración de los beneficios, norma subjetiva, actitud hacia la piratería, habilidades), utilizando el método de estimación ML Robust. La intención de descarga de música y la capacidad técnica no se incluyeron dado que se trataba de dos escalas con un único ítem cada una.

Para conseguir la validez convergente fueron eliminados aquellos ítems cuyas cargas factoriales eran no significativas o inferiores a 0.60 (Bagozzi y Baumgartner, 1994; Bagozzi y Yi, 1988) o para los que el test de los multiplicadores de Lagrange sugería cargas significativas sobre un factor distinto del que eran indicadores (Hatcher, 1994). Ello supuso eliminar dos ítems de la escala "Percepción de las consecuencias del comportamiento", cinco ítems de la escala "Valoración de los beneficios de la descarga de música", un ítem de la escala "Norma subjetiva" y un ítem de la escala "Actitud hacia la descarga de música".

El modelo resultante se refleja en el cuadro 2. El valor de la Chi-cuadrado es significativa $\left(a^{2}=319,545 ; g l=105 ; p<0,01\right)$, y el resto de indicadores específicos muestran un ajuste razonable (BBNFI=0,940; $B B N N F I=0,946$; $C F|=0,958 ;| F \mid=0,959 ; R M S E A=0,04)$. Las cargas factoriales significativas y superiores en su totalidad a 0,60 (Bagozzi y Yi, 1988) confirman la validez convergente del instrumento propuesto. 
TABLA 2

\section{INSTRUMENTO DE MEDIDA: FIABILIDAD Y VALIDEZ CONVERGENTE}

\begin{tabular}{|c|c|c|c|c|c|c|}
\hline Factor & Indicador & $\begin{array}{c}\text { Carga } \\
\text { Estandarizada }\end{array}$ & Valor t & $\begin{array}{l}\text { a de Cronba- } \\
\text { ch }\end{array}$ & $\begin{array}{l}\text { Fiabilidad } \\
\text { Compuesta }\end{array}$ & $\begin{array}{c}\text { Varianza extraí- } \\
\text { da promedio } \\
\text { AVE }\end{array}$ \\
\hline \multirow{2}{*}{$\begin{array}{l}\text { F1. Percepción } \\
\text { consecuencias } \\
\text { comportamiento }\end{array}$} & CON3 & 0,856 & $20,69^{* *}$ & \multirow{2}{*}{0,717} & \multirow{2}{*}{0,7012} & \multirow{2}{*}{0,5368} \\
\hline & CON4 & 0,684 & $16,10^{* *}$ & & & \\
\hline \multirow{4}{*}{$\begin{array}{l}\text { F2. Valoración de las } \\
\text { consecuencias del } \\
\text { comportamiento }\end{array}$} & VC01 & 0,761 & $32^{\prime} 56^{* *}$ & \multirow{4}{*}{0,813} & \multirow{4}{*}{0,8502} & \multirow{4}{*}{0,5881} \\
\hline & VCO2 & 0,682 & $31,10^{* *}$ & & & \\
\hline & VCO3 & 0,773 & $33,30^{* *}$ & & & \\
\hline & VCO4 & 0,843 & $43,32^{* *}$ & & & \\
\hline \multirow{4}{*}{$\begin{array}{l}\text { F3. Valoración de los } \\
\text { beneficios de la descarga } \\
\text { de música }\end{array}$} & VBE3 & 0,601 & $12,01^{* *}$ & \multirow{4}{*}{0,702} & \multirow{4}{*}{0,7006} & \multirow{4}{*}{0,5920} \\
\hline & VBE4 & 0,706 & $20,37^{* *}$ & & & \\
\hline & VB9 & 0,610 & $13,36^{* *}$ & & & \\
\hline & VB10 & 0,623 & $15,19^{* *}$ & & & \\
\hline \multirow{2}{*}{ F4. Norma subjetiva } & NOR1 & 0,756 & $24,82^{* *}$ & \multirow{2}{*}{0,768} & \multirow{2}{*}{0,7494} & \multirow{2}{*}{0,5994} \\
\hline & NOR2 & 0,792 & $24,33^{* *}$ & & & \\
\hline \multirow{3}{*}{$\begin{array}{l}\text { F5. Actitud hacia la } \\
\text { descarga de música }\end{array}$} & ACT1 & 0,726 & $25,17^{* *}$ & \multirow{3}{*}{0,811} & \multirow{3}{*}{0,8212} & \multirow{3}{*}{0,5500} \\
\hline & ACT2 & 0,757 & $23,96^{* *}$ & & & \\
\hline & ACT4 & 0,650 & $18,80^{* *}$ & & & \\
\hline \multirow{2}{*}{$\begin{array}{l}\text { F6. Habilidades para la } \\
\text { descarga de música }\end{array}$} & HAB1 & 0,951 & $30,98^{* *}$ & \multirow[t]{2}{*}{0,937} & \multirow{2}{*}{0,9015} & \multirow{2}{*}{0,8211} \\
\hline & HAB2 & 0,859 & $29,85^{* *}$ & & & \\
\hline
\end{tabular}

Nota. ${ }^{* *} p<.01 ;{ }^{*} p<.05$

Fuente: elaboración propia.

Respecto a la fiabilidad, todos los a de Cronbach (Cronbach, 1951) son superiores al valor recomendado de 0,7 (Churchill, 1979). En el cuadro 2 se ofrece asimismo la fiabilidad compuesta, constatándose también que su valor es superior, para todos los factores, al valor recomendado de 0,7 (Fornell y Larcker, 1981). La última columna ofrece la varianza promedio extraída, que es un indicador de la varianza capturada por un factor respecto a la varianza debida al error de medida (Fornell y Larcker, 1981). Este indicador muestra que para todos los factores se supera el valor de 0,5 que es el recomendado por Fornell y Larcker (1981). Tomados en su conjunto, todos estos in- dicadores demuestran evidencias suficientes de fiabilidad del instrumento de medida.

Para analizar la validez discriminante se han seguido dos procedimientos: (a) comprobar que el intervalo de confianza en la estimación de la correlación entre cada par de factores no incluye el valor 1 (Anderson y Gerbing, 1988) y (b) que la varianza promedio extraída para cada factor sea superior al cuadrado de la correlación entre cada par de factores (Fornell y Larcker, 1981). El cuadro 3 muestra los resultados, lo que permiten afirmar la validez discriminante del instrumento de medida propuesto. 
TABLA 3

INSTRUMENTO DE MEDIDA: VALIDEZ DISCRIMINANTE

\begin{tabular}{|r|r|r|r|r|r|r|}
\hline & F1 & F2 & F3 & F4 & F5 & F6 \\
\hline F1 & .53 & $0,064^{* *}$ & $0,0009 n s, 166^{* *}$ & $0,129^{* *}$ & $0,041^{* *}$ \\
\hline F2 & {$[-.192 ;-.328]$} & .58 & $0,019^{* *}$ & $0,051^{* *}$ & $0,106^{* *}$ & $0,016^{* *}$ \\
\hline F3 & {$[.004 ;-.156]$} & {$[.946 ;-.534]$} & .59 & $0,008^{* *}$ & $0,061^{* *}$ & $0,131^{* *}$ \\
\hline F4 & {$[.985 ;-.245]$} & {$[.403 ;-.917]$} & {$[-.0718 ;--.231]$} & .59 & $0,170^{* *}$ & $0,057^{* *}$ \\
\hline F5 & {$[.372 ;-.899]$} & {$[.991 ;-.279]$} & {$[.979 ;-.366]$} & {$[-.384 ;-.516]$} & .55 & $0,219^{* *}$ \\
\hline F6 & {$[-.092 ;-.224]$} & {$[.737 ;-.503]$} & {$[.952 ;-.134]$} & {$[.487 ;-.833]$} & {$[.977 ;-.140]$} & .82 \\
\hline
\end{tabular}

Nota. ${ }^{* *} p<.01 ;{ }^{*} p<.05 ;$ ns no significativa

Debajo de la diagonal: intervalo de confianza para la correlación entre factores. Diagonal: varianza extraída. Sobre la diagonal: cuadrado de la correlación estimada entre los factores

Fuente: elaboración propia.

Evaluadas las propiedades psicométricas del instrumento de medida, se procedió a estimar el modelo estructural recogido en la figura 1 que sintetiza las hipótesis planteadas a partir de los resultados de la dinámica realizada. Tras realizar la identificación del modelo, éste fue estimado por el método robusto, utilizando EQS 6.1.

Los resultados de la estimación del modelo inicial mostraron un ajuste razonable en el que todas las relaciones planteadas resultaban sig- nificativas; no obstante, el test de Lagrande planteaba la inclusión de una nueva relación, no tenida en cuenta en el modelo inicial pero que, tras valorarla, se planteaban como lógica: se trata de la influencia entre la disponibilidad técnica para descargar música y la percepción de que se poseen habilidades para ello. Planteada esta modificación, el modelo revisado (cuadro 4) muestra un ajuste adecuado (a2 (138 gl) $=509,013(\mathrm{p}<0,01) ; \mathrm{BBNFI}=, 917 ; \mathrm{BBNNFI}=, 923$; $\mathrm{CFI}=, 938 ; \mathrm{IFI}=, 938 ; \mathrm{RMSEA}=, 046)$.

TABLA 4

\section{CONTRASTE DE HIPÓTESIS MODELO REVISADO}

\begin{tabular}{|c|c|c|}
\hline HIPÓTESIS & $\begin{array}{c}\text { Carga } \\
\text { Estandarizada }\end{array}$ & Valor t \\
\hline H1: Actitud hacia la descarga de música no autorizada de Internet $\rightarrow(+)$ Intención de descargar música & $0.594^{* *}$ & 14.547 \\
\hline H2: (reencias sobre las consecuencias negativas de la descarga de música no autorizadaà(-) Actitud hacia la descarga de música & $-0.165^{* *}$ & -3.738 \\
\hline H3: Valoración de los beneficios de la descarga de música no autorizada $\rightarrow(+)$ Actitud hacia la descarga de música & $0.288^{* *}$ & 6.144 \\
\hline H4: Importancia que se atribuye a las consecuencias negativas de la descarga de música no autorizada $\rightarrow$--) Actitud hacia la descarga de música & $-0.174^{* *}$ & -4.734 \\
\hline $\begin{array}{l}\text { H5: Percepción sobre la opinión negativa de los demás en relación a la descarga de música no autorizada de Internet (norma subjetiva) } \\
\rightarrow(-) \text { Actitud hacia la descarga de música. }\end{array}$ & $-0.291^{* *}$ & -6.073 \\
\hline $\begin{array}{l}\text { H6: Percepción sobre la opinión negativa de los demás en relación a la descarga de música no autorizada de Internet (norma subjetiva) } \\
\rightarrow(-) \text { Intención de descargar música }\end{array}$ & $-0.083^{* *}$ & -2.419 \\
\hline H7: Percepción de poseer las habilidades necesarias para descargar música no autorizada de Internet $\rightarrow(+)$ Intención de descargar música & $0.306^{* *}$ & 8.859 \\
\hline H8: Disponibilidad técnica para descargar música no autorizada de Internet $\rightarrow(+)$ Intención de descargar música & $0.150^{* *}$ & 4.563 \\
\hline $\begin{array}{l}\text { Nueva relación: Disponibilidad técnica para descargar música no autorizada de Internet } \rightarrow(+) \text { Percepción de poseer las habilidades } \\
\text { necesarias para descargar música }\end{array}$ & $0.478^{* *}$ & 14.237 \\
\hline
\end{tabular}

Nota. ${ }^{* *} \mathrm{p}<.01 ;{ }^{*} \mathrm{p}<.05$; ns no significativa

Fuente: elaboración propia 
Según los resultados obtenidos se constata cómo la actitud hacia la descarga de música es la variable que más influye en la intención de descargar música no autorizada de Internet, seguida de la percepción que posea el individuo sobre las habilidades que tiene para desarrollar tal comportamiento y las disponibilidades de capacidad técnica. A su vez, la capacidad técnica determina las habilidades del individuo en la descarga de música: como es lógico, cuanta más capacidad técnica se posee, más se irán desarrollando las habilidades del individuo y, como consecuencia, así lo percibirá. Además, aunque con una influencia muy débil, la norma subjetiva, es decir, lo que el individuo crea que piensan los demás acerca de la descarga de música no autorizada, también influirá en su intención de descargar.

Por su parte, la actitud hacia la descarga viene condicionada, en primer lugar, por la norma subjetiva, seguida de la percepción de los beneficios que el individuo atribuye a la descarga de música. Le siguen la importancia, que a nivel personal, el individuo atribuye a las consecuencias negativas de la descarga de música, y en último lugar, la propia percepción de dichas consecuencias negativas.

\section{CONCLUSIONES E IMPLICACIONES DE GESTIÓN}

No cabe duda de que la piratería musical es un fenómeno cada vez más extendido y que, como se ha puesto de manifiesto en este estudio, no es percibido como algo incorrecto o negativo por parte de los individuos. A ello se une la disponibilidad técnica para descargar música, dado que los avances tecnológicos están favoreciendo que los individuos, a edades cada vez más tempranas, tengan acceso a ordenador, grabadora de Cd/Dvd, banda ancha a precios cada vez más asequibles, lo que hace también que las habilidades necesarias para el uso de estos aparatos y programas necesarios sea cada vez más fácil para cualquiera.

Son diversas las medidas que se han desarrollado para luchar contra el fenómeno de la piratería, pero no han dado los resultados que, en un principio, se esperaban. Quizás el no conocer exactamente qué es lo que determina el desarrollo de dicho comportamiento ha favorecido tales resultados. En este sentido, la Teoría del Comportamiento Planeado (Ajzen, 1991) ofrece un marco de referencia a partir del cual considerar qué puede determinar el desarrollo de un determinado comportamiento. Esta teoría, que ha sido especialmente útil en el ámbito de conductas vinculadas con connotaciones éticas, como es el caso de la descarga de música de Internet, plantea que el desarrollo de un determinado comportamiento está determinado por la intención de desarrollarlo. A su vez, dicha intención está condicionada por la actitud hacia el comportamiento en cuestión y lo que se denomina norma subjetiva, es decir, lo que el individuo percibe sobre lo que la gente opina en relación al comportamiento estudiado. En última instancia, según esta teoría, la actitud vendrá determinada por la valoración que haga el individuo de las consecuencias, positivas y negativas, de tal comportamiento, dado que se supone que el comportamiento humano es bastante racional y valora, pues, las consecuencias de sus actos. Partiendo de estas relaciones, y considerando la flexibilidad que ofrece la Teoría del Comportamiento Planeado para incorporar variables no consideradas en la base inicial de la Teoría, en el presente estudio se ha estimado oportuno analizar asimismo, como antecedente de la actitud hacia la descarga, la importancia que atribuye el in- 
dividuo, a nivel personal, a las consecuencias negativas percibidas por la descarga de música: si tenemos en cuenta que, a priori, los beneficios que el individuo pueda percibir por al descarga de música son más bien beneficios personales, pero las consecuencias negativas repercuten en otros (no a nivel personal), será interesante considerar hasta qué punto tales consecuencias en "otros" le importan al consumidor.

Todas las variables analizadas en este estudio han resultado relevantes y significativas en la explicación de la intención de descargar música gratuita de Internet, especialmente la actitud hacia la descarga de música. A partir de estos resultados se pueden plantear tres grandes tipos de actuaciones conjuntas.

En primer lugar, y considerando que de los tres antecedentes considerados de la intención de descargar música (capacidad técnica, habilidades necesarias, y actitud hacia la descarga) sólo es posible influir en uno de ellos, la actitud, será necesario el desarrollo de acciones en torno a esta variable. Como se ha constatado, en dicha actitud influye lo que los demás opinen del hecho de descargar música, la percepción de las consecuencias (negativas y positivas) de la descarga, y la importancia que el individuo atribuya a las repercusiones negativas de la descarga; consideramos pues necesario el desarrollo de acciones de comunicación, centradas sobre todo en publicidad, y coloquios y seminarios en colegios y universidades. Dichas acciones deberían ir encaminadas a reforzar el valor de poseer los originales, y a mostrar realmente las consecuencias negativas del comportamiento aquí analizado, en términos de pérdidas económicas y puestos de trabajo, y el consecuente cierre de empresas, menos oportunidades para los artistas jóvenes,..., con el propósito de hacer más consciente al individuo de las consecuencias negativas que su comportamiento desencadena, tratando de influir, también, en la importancia personal atribuida a dichas consecuencias. De esta forma, también se estaría influyendo en lo que se ha denominado norma subjetiva, dado que podría llevar a individuo a percibir que la opinión de "los demás" respecto a la descarga de música no es positiva, a la vista de las consecuencias negativas conocidas.

El eje de las campañas de comunicación propuestas debería elaborarse con mucho cuidado, dado que el coste del desarrollo de dichas campañas es alto y, además, ya se han desarrollado campaña de comunicación pero sin mucho éxito.

Una segunda línea de actuación a proponer sería el desarrollo de estrategias de precio. Dado que los individuos consideran que los CDs son caros, y que la motivación económica es muy relevante cuando se habla de piratería musical, se podrían ofrecer precios más bajos, justificados por una presentación del Cd más austera. Quizás una parte de la población que actualmente descarga música no autorizada de Internet estaría dispuesta a pagar precios más bajos por adquirir música legal.

En último lugar pero quizás con una importancia mucho más detacada, pensando en un futuro más a medio plazo, y teniendo en cuenta la dificultad/imposibilidad de volver a los patrones de consumo de música previos a la revolución digital en la industria musical, creemos que se deben introducir nuevas medidas de gestión y marketing, y especialmente desarrollar nuevas formas de venta. Tal y como apunta Alonso (2010), "el modelo de la industria musical tiene que evolucionar o 
morirá definitivamente, pues la tecnología ha hecho que los modelos de negocio basados en las dificultades de distribución salten por los aires". Este cambio debe ir más allá de las medidas planteadas, hace algo más de una década, por Gopal y Sanders (1997) dirigidas a luchar contra la piratería de manera preventiva y disuasoria y que todavía siguen siendo el leitmotiv de la Federación Internacional de la Industria Discográfica, gobiernos y colectivos de artistas, entre otros. Es cierto que la industria de la música ya ha empezado a actuar en este ámbito y que son numerosas las nuevas decisiones de venta que se han venido diseñando y aplicando. En este ámbito, se destacan aquellas medidas más relevantes e incluso se sugieren otras nuevas que abarcarían tres ámbitos distintos: producto, evento, y nuevos canales.

En términos de producto, la música en sí misma, ha habido pocas innovaciones en los últimos años por parte de las discográficas, que han lanzado y reeditado sus catálogos ya existentes con grandes éxitos y recopilaciones. En este sentido, debería de ampliarse la oferta de música digital así como de los estilos de música minoritarios (clásica, folk, étnica,...).

Pero el producto no sólo se vincula al grupo y las canciones, es decir, al producto central, sino también a todos aquellos elementos auxiliares, que conforman el denominado producto aumentado, y que hacen la oferta más atractiva, notoria y diferenciada. La explotación y venta online de productos vinculados a los grupos de una discográfica, como camisetas, gorras, llaveros y demás artículos de merchandising puede permitir a las casas de discos generar ingresos vías otras fuentes. De igual modo, la inclusión de elementos que no puedan incorporarse en copias gratuitas o ilegales como packings más atractivos y cuida- dos o invitaciones a eventos, entre otros, puede suponer no sólo un aliciente para aquellos seguidores de un grupo sino ciertos ingresos para una compañía.

Un segundo grupo de medidas se vincula a los eventos. Así, una medida necesaria es el aumento del número de actuaciones en directo de los distintos grupos y la gestión de dichas giras. Son ya varias las discográficas que, a la vista del dinamismo de este mercado, intentan incluir en los contratos con nuevos artistas la participación en estas nuevas guías de negocio (conciertos y merchandising) y además tratan de incluirlos en la renovación a los artistas existentes en catálogo. Son los denominados contratos 360 grados, o todo incluido. Contratos donde no sólo se incluyen derechos sobre música grabada sino también de explotación de conciertos, el management y merchandising (The Economist, 2007). El directo es además un aspecto esencial para los artistas. Supone junto a la calidad de su música el criterio más importante para ser seleccionados y producidos por una discográfica (Zwaan y ter Bogt, 2009) y con ello poder alcanzar el éxito.

Finalmente y en relación a los nuevos formatos de venta, resulta relevante aumentar el número de soportes, es decir, de dispositivos digitales a partir de los cuales poder consumir música. A los ya consolidados PC, Mac, reproductores mp3 y mp4, y los teléfonos móviles, se empiezan a considerar las consolas y las suscripciones a bibliotecas, entre otras posibilidades. Se trata de hacer disponible la música a través de nuevos canales, bien de pago o gratuitos, conllevando menores costes de transacción para el consumidor, como evitar el tiempo invertido en la asistencia a un punto de venta para la adquisición de un álbum de música. 
De igual forma la aparición de los nuevos canales o plataformas para consumir música han supuesto un cambio no sólo en la forma de distribuir y consumir música sino también en las pautas de financiación. Así, son varios los nuevos modelos de venta y financiación surgidos en la industria de la música grabada. Desde el modelo de pago por descarga (digital download), bien de un álbum o una canción, al modelo de suscripción (streaming suscription) por el que se realiza el pago de una cuota mensual para escuchar música pero sin descarga. Paralelamente, aparecen las redes sociales, que si bien permiten promocionar un producto conllevan una mayor dificultad para controlarlo. La aparición de estos nuevos canales de distribución de música condiciona entre otros, los objetivos promocionales de artistas y discográficas. Si hace una década, la música debía estar en establecimientos comerciales de música, hoy en día es prioritario que esté disponible en plataformas de streaming. Si los videoclips debían emitirse por canales de televisión, ahora es obligado estar en plataformas como Youtube. Las diferentes emisoras de radio, donde debían sonar los discos, ya no son tan relevantes pues se persiguen campañas más completas y complejas a través de redes sociales como Facebook, tuenti y otras.

Un último bloque de medidas dentro de esta línea recoge las alianzas estratégicas, como el replanteamiento de acuerdos con emisoras de radio, pues en definitiva son las discográficas quienes proveen a las radios del contenido que necesitan para atraer una audiencia que, a su vez, los anunciantes desean alcanzar. En esta línea, las alianzas podrían establecerse también con las compañías telefónicas, dado el aumento de demanda de música a través de los teléfonos móviles y el papel que va a ju- gar el teléfono en los próximos años, así como alianzas con páginas web. Se trata, en definitiva, de crear nuevos canales de distribución.

No hay duda de que los hábitos de consumo de música del individuo han cambiado en los últimos años gracias a las nuevas tecnologías $y$, especialmente, a Internet. Consideramos que es muy difícil, si no imposible, parar este proceso, por lo que el sector de la industria musical debe plantarse nuevos retos y reconsiderar sus propias estrategias de negocio.

Aunque son varias las variables analizadas en este estudio como determinantes de la intención de descargar música no autorizada de Internet, planteamos como futuras líneas de investigación seguir ahondando en el análisis de nuevas variables antecedentes con el propósito de buscar vías alternativas de actuación para las discográficas ante un comportamiento tan extendido como es el de la descarga de música no autorizada.

\section{REFERENCIAS}

Ajzen, I. (1985). From intentions to behaviour: a theory of planned behaviour, en I.J.K.J.B. (eds.), Action-Control: From Cognition to Behaviour- Heidelberg: Spinger pp. $11-39$.

Ajzen, I. (1991): The theory of planned behaviour. Organizational Behaviour and Human Decision Processes, 50 (1), pp. 179-211.

Ajzen, I. \& Fishbein, M. (1980). Understanding attitudes and predicting social behavior Englewood Cliffs, N.J.: Prentice-Hall.

Ajzen, I. \& Madden, T. (1986): Perceived goal-directed behaviour: Attitudes, dimensions and perceived behavioural control. Journal of Experimental Social Psychology, 22, pp. 453-474.

Alonso, J. (2010, 24 de Diciembre). "Un cambio imparable". El País. Recuperado el 24/12/2010 de:

http://www.elpais.com/articulo/cultura/cambio/imparable/elpepicul/20101224elpepicul_2/Tes 
Anderson, J.C. \& Gerbing, D.W. (1988): Structural equation modelling in practice: a review and recommended two-step approach. Psychological Bulletin, 103, pp. 411-23.

Bagozzi, R.P. (1981): Evaluating structural equations models with unobservable variables and measurement error: a comment. Journal of Marketing Research, 18 (August), pp. 375-381.

Bagozzi, R.P. \& Yi, Y. (1988): On the evaluation of structural equations models. Journal of the Academy of Marketing Science, 16 (1), pp. 74-94.

Bagozzi, R.P. \& Baumgartner, H (1994): The evaluation of structural equation models and hypothesis testing. En Bagozzi, R. (Eds), Principles of Marketing Research Cambridge, MA: Blackwell Publishers, pp.386-422.

Bailey, A.A. (2006): Retail employee theft: a theory of planned behaviour perspective. International Journal of Retail \& Distribution Management, 34 (11), pp. 802816.

Bentler, P.M. (1989). EQS structural equations program manual. Los Angeles: BMDP Statistical Software.

Chiou, J., Huang, C. \& Lee, H. (2005): The antecedents of music piracy attitudes and intentions. Journal of Business Ethics, 57, pp. 161-174.

Chiu, R. (2003): Ethical judgment and whistle Blowing intention: examining the moderating role of locus of control. Journal of Business Ethics, 43, pp. 65-74.

Churchill, G.A. (1979): A Paradigm for Developing Better Measures of Marketing Constructs. Journal of Marketing Research, 16 (1), pp. 64-73.

Cronan, T.P. \& Al-Rafee, S. (2008): Factors that influence the intention to pirate software and media. Journal of Business Ethics, 78 (April), pp., 527-545.

Cronbach, L. S. (1951): Coefficient alpha and the internal structure of test. Psychometrika, 16 (3), pp. 297-334.

D'Astous, A., Colbert, F. \& Montpetit, D. (2005): Music piracy on the Web - How effective are the Anti-piracy arguments? Evidence from the theory of Planned behaviour. Journal of Consumer Policy, 28, pp. 289-310.

Dubinsky, A.J. \& Loken, L. (1989): Analysing ethical decision making in marketing. Journal of Business Research, 19 (2), pp. 83-108.
Fishbein, M. \& Ajzen, I. (1975). Belief, Attitude, Intention, and Behaviour: an introduction to Theory and Research Reading, M.A.: Addison-Wesley.

Flannery, B.L. \& May, D.R. (2000): Environmental ethical decision making in the U.S. metal-finishing industry. Academy of Management Journal, 43 (4), pp. 642-662.

Fornell, C. \& Larcker, D.F. (1981): Evaluating structural equation models with unobservable variables and measurement error. Journal of Marketing Research, 18 (February), pp. 39-50.

Gopal, R. \& Sanders, L. (1997): Global software piracy: You can't get blood out of your turnip. Communications of the ACM, 43 (9), pp. 82-89.

Gopal, R.D., Sanders, G.L., Bhattacharjee, S., Agrawal, M. \& Wagner, S.C. (2004): A behavioural model of digital music piracy. Journal of Organizational Computing and Electronic Commerce, 14 (2), pp. 89

Gupta, P.B., Gould, S.J. \& Pola, B. (2004): To pirate or not to pirate: a comparative study of the ethical versus other influences on the Consumer's software acquisition-mode decision. Journal of Business Ethics, 55, pp. 255-274.

Hatcher, L. (1994). A step-by-step approach to using the SAS system for factor analysis and structural equation modelling. Cary, NC: SAS Institute Inc.

Longsdon, J.M., Thompson, J.K. \& Reid, R.A. (1994): Software piracy: is it related to level of moral judgement? Journal of Business Ethics, 13 (11), pp. 849-857.

Norusis, M. (1993). SPSS for windows professional statistics. Ed. SPSS Inc.

Nunnally, J.C. (1978). Psychornetric Theory. 2d ed. New York: McGraw-Hill.

Parker, D., Manstead, A.S.R., Stradling, S.G., Reason, J.T. \& Baxter, J.S. (1992): Intention to commit driving violations: an application of the theory of planned behaviour. Journal of Applied Psychology, 77 (1), pp. 94-101.

SGAE (2010). Anuario Sgae de las Artes Escénicas, Musicales y Audiovisuales 2010. Recuperado el 17-2-2011 de:

http://www.sgae.es/recursos/documentacion/ANUARIO_2010.pdf 
RNA Revista Nacional de Administración

The Economist (2007, 5 de Julio). A change of tune. The Economist. Recuperado el 10-7-2007 de: http://www.economist.com/node/9443082?story_id=9443082

Tonglet, M. (2002): Consumer misbehaviour: an exploratory study of shoplifting. Journal of Consumer Behaviour, 1 (4), pp. 336-354.

Zwaan, K. \& ter Bogt, T.F.M. (2009): Breaking into the popular record industry: an insider's view on the career entry of pop musicians. European Journal of Communication, 24, pp. 89-101.

Recibido: 18-11-2011

Aceptado: 10-02-2012 